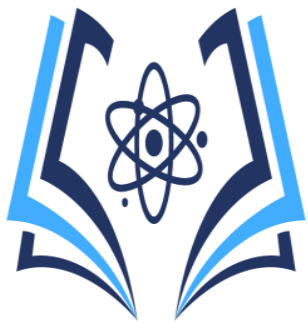

\title{
Bioseguridad para enfrentar la COVID 19 en Ecuador
}

\author{
Biosecurity to face COVID 19 in Ecuador
}

Bioseguridad para enfrentar la COVID

\author{
María del Rosario Herrera Velázquez. PhD \\ Universidad Estatal del Sur de Manabí, mariarosario.herrera@unesum.edu.ec; vehemari@gmail.com
}

Contacto: mariarosario.herrera@unesum.edu.ec

Recibido: 25-02-2020

Aprobado: 23-06-2020

\section{Resumen}

Estudiantes del segundo semestre de la carrera de enfermería, como parte del proceso de formación, desarrollaron una investigación bibliográfica, de carácter multidisciplinario, cualitativo y descriptivo, en el marco del Proyecto Integrador de Saberes (PIS), con el objetivo de sintetizar las medidas de bioseguridad ante el Covid-19 establecidas por el Ministerio de Salud Pública de Ecuador. Se utilizó como motor de búsqueda el google académico, consultando bases de datos como SCielo y LILACS, utilizando los descriptores covid-19, fitoterapia y medidas de bioseguridad. Se logró sistematizar aspectos microbiológicos del virus, características, signos y síntomas de la infección, modo de transmisión, complicaciones frecuentes, medidas de prevención ante la propagación del virus, así como el uso de terapias complementarias como lo son los fitofármacos, haciendo énfasis en reportes científicos que avalen su actividad, composición química, indicación, interacciones, toxicidad y reacciones adversas, entre otras. La información obtenida sobre el covid-19 indica que es una enfermedad altamente transmisible y la única metodología para prevenir la infección es evitar la exposición al virus, ya que aún no existe vacuna ni tratamiento específico para prevenir o tratar la infección que causa. Las medidas de prevención que utiliza el personal de salud son primordiales para prevenir la exposición a riesgos y la importancia de acatar las medidas de bioseguridad descritas por el Ministerio de Salud Pública es un complemento fundamental para la disminución de contagios. Como terapia complementaria a las infecciones respiratorias provocadas por coronavirus, se puede utilizar

fitofármacos que contengan extractos de plantas que ayuden a disminuir los síntomas y que tengan algún respaldo científico en cuanto a su eficacia farmacológica.

Palabras clave: coronavirus, enfermería, fitoterapia

Abstrat

Students of the second semester of the nursing career, as part of the training process, developed a bibliographic research, multidisciplinary, qualitative and descriptive, within the framework of the Integrative Knowledge Project (PIS), with the aim of synthesizing the measures of biosecurity against Covid-19 established by the Ministerio de Salud Pública de Ecuador. The academic google was used and consulting databases such as SCielo and LILACS, using the descriptors covid-19, phytotherapy and biosafety measures. It was possible to systematize microbiological aspects of the virus, characteristics, signs and symptoms of the infection, mode of transmission, frequent complications, preventive measures against the spread of the virus, as well as the use of complementary therapies such as phytotherapy, emphasizing reports scientists who endorse its activity, chemical composition, indication, interactions, toxicity and adverse reactions, among others. The information obtained on covid-19 indicates that it is a highly communicable disease and the only methodology to prevent infection is to avoid exposure to the virus, since there is still no vaccine or specific treatment to prevent or treat the infection it causes. The prevention measures used by health personnel are essential to prevent exposure to risks and the importance of complying with the biosecurity measures described by the Ministerio 


\section{HIGIA DE LA SALUD}

de Salud Pública (MSP) is a fundamental complement to reduce infections. As a complementary therapy to respiratory infections caused by coronavirus, phytopharmaceuticals that contain plant extracts that help reduce symptoms and that have some scientific support in terms of their pharmacological efficacy can be used.

Keywords: coronavirus, nursing, phytotherapy Introducción

El brote de la enfermedad infectocontagiosa denominada Covid-19, ha trastocado la vida de los humanos en el planeta, con repercusiones en todos los ámbitos y con una propagación y mortalidad sin precedentes en la historia actual. Esta enfermedad se dio en Wuhan una ciudad de China, el lugar donde se rastrearon los primeros casos fue en un mercado de mariscos y animales de la misma ciudad, algunos investigadores manifiestan que esta cepa pudo provenir de murciélagos, es por ello, que existe la posibilidad que desde ahí el virus se expandiera a los humanos (1).

El mundo actualmente está expuesto a la enfermedad del Covid-19, que afecta la calidad de vida normal que desarrollaban las personas en sus actividades cotidianas como son los estudios, trabajo, momentos de distracción, eventos religiosos, etc. Así mismo, debemos mencionar algunas de las sintomatologías que esta ocasiona como lo es la fiebre, tos y dificultad para respirar entre otras.

En el Ecuador el virus se ha expandido rápidamente y las cifras de contagios crecen cada día, por otro lado, las cifras oficiales de fallecidos igualmente aumenta (2). Debido a esta situación, las autoridades correspondientes se han visto en la obligación de tomar medidas de protección para todos los ciudadanos. Una de las normas de bioseguridad que se implementó por la existencia de la pandemia fue el confinamiento social para poder salvaguardar la salud de las personas.

Teniendo en cuenta lo planteado, este trabajo tiene como objetivo sintetizar las medidas de bioseguridad del Covid-19 establecidas por el Ministerio de Salud Pública de Ecuador. Para alcanzar el mismo, se trazaron como tareas de investigación:

Describir las características microbiológicas del virus, mecanismo de transmisión, manifestaciones clínicas y complicaciones.

Identificar las medidas de prevención que utiliza el personal de enfermería para evitar el riesgo de contagio del Covid-19.

Determinar si el equipo multidisciplinario ha recibido capacitaciones continuas sobre el manejo de pacientes con Covid-19.

Describir fitofármacos que se comercializan en Ecuador, para tratar enfermedades respiratorias provocadas por coronavirus, atendiendo a su uso,
ISSN 2773-7705

Periodo. Enero - Junio 2020

Vol. 2, Nro. 1, Publicado 2020-06-30

forma farmacéutica, composición química, vía de administración, dosis, RAM, toxicidad e interacciones medicamentosas.

\section{Materiales y métodos}

Se desarrolló una investigación bibliográfica, de carácter multidisciplinario, cualitativo y descriptivo, analizando la información recopilada de manera tal que los estudiantes pudieron reforzar y ampliar los conocimientos sobre el tema investigado. Se consultaron bases de datos internacionales disponibles en la web del conocimiento, se describió de manera clara y concreta la información de este proyecto para un mejor conocimiento sobre el Covid-19 en estos tiempos de emergencia sanitaria.

Se utilizó como motor de búsqueda el google académico, se consultaron bases de datos como SCielo y LILACS, utilizando los descriptores covid-19, fitoterapia y medidas de bioseguridad. Se obtuvieron más de 1000 artículos y publicaciones diversas, las cuales se analizaron y filtraron teniendo como criterio el idioma (español), publicaciones de América Latina, América del Sur y las publicaciones de la OMS y OPS. Siendo así, se analizaron 30 publicaciones, las cuales se utilizaron para elaborar el informe. Se tuvieron en cuenta aspectos microbiológicos del virus, características, signos y síntomas de la infección, las medidas de prevención ante la propagación del virus, así como el uso de terapias complementarias como lo son los fitofármacos, haciendo énfasis en las características principales en cuanto a composición, indicación, interacciones, toxicidad y reacciones adversas, entre otras.

\section{Resultados}

Características microbiológicas del virus

Los coronavirus son un grupo amplio de virus, y deben su nombre a la apariencia de una corona en su parte externa, causan infección en humanos y en una variedad de animales, incluidos aves y mamíferos. En sus inicios es considerada una enfermedad zoonótica, lo que significa que puede transmitirse de animales a humanos, aunque luego se transmita entre humanos.

Estructuralmente, los coronavirus son virus esféricos con un diámetro de 100-160 nm que están envueltos en ARN positivo no segmentado entre 26 y 32 kilobases de longitud; pertenecen a la familia Coronaviridae, del orden Nidovirales. Esta subfamilia comprende cuatro géneros: Alphacoronavirus,

Betacoronavirus, Gammacoronavirus y Deltacoronavirus según su estructura genética (3).

Los alfacoronavirus y betacoronavirus solo infectan a los mamíferos y suelen ser responsables de infecciones respiratorias en humanos, aunque raras veces también pueden causar trastornos hepáticos y neurológicos, y gastroenteritis en animales. El nuevo coronavirus 


\section{HIGIA DE LA SALUD}

SARS-CoV-2 es el responsable de la pandemia actual $\mathrm{y}$ ha provocado una crisis sanitaria $\mathrm{y}$ económica como nunca antes (4).

\section{Mecanismo de transmisión}

El nuevo coronavirus SARS-CoV-2 (síndrome respiratorio agudo grave) se transmitió en primer lugar de animales a humanos, pero enseguida se comprobó que existía la transmisión entre personas. La vía de contagio entre humanos se produce a través del contacto directo con las secreciones respiratorias que se producen sobre todo al respirar, toser o estornudar; también puede darse mediante contacto con las manos y otros objetos contaminados por estas secreciones, y tras tocarse la boca, la nariz o los ojos. El virus puede ser viable en el aire, por lo que también es posible la transmisión aérea por aerosoles, aunque de manera restringida, no en espacios abiertos y principalmente en entornos sanitarios (5). Finalmente, el virus también se ha detectado en heces, pero pese a algunos indicios de posible infección gastrointestinal, hasta el momento no se ha demostrado la transmisión fecal-oral. La carga viral, que determina en parte la capacidad de transmisión, es mayor durante la primera semana de enfermedad, y se sabe que personas asintomáticas también podrían contagiarlo, algo que podría estar relacionado con altas concentraciones del virus en la garganta (6).

\section{Manifestaciones clínicas}

Las manifestaciones clínicas frecuentes incluyen fiebre, tos seca, odinofagia, cefalea, astenia, mialgia y dificultad respiratoria. Hasta el momento, las manifestaciones cutáneas relacionadas con Covid-19 solo se han descrito en la población infantil previamente asintomáticos, que se manifiesta como un síndrome hiperinflamatorio con afectación multiorgánica, similar al síndrome shock de la enfermedad de Kawasaki (7).

Los signos y síntomas de la enfermedad Covid19 , pueden aparecer entre dos y 14 días después de la exposición al virus. Los signos y síntomas más comunes pueden incluir fiebre, tos y fatiga. Los primeros síntomas de Covid-19 pueden incluir pérdida del gusto o del olfato. Esta lista no incluye todos los posibles signos y síntomas, se han informado otros síntomas menos comunes, como erupción cutánea, náuseas, vómitos y diarrea. Los niños tienen síntomas similares a los de los adultos y generalmente tienen una enfermedad leve (8).

\section{Complicaciones}

Las complicaciones descritas asociadas con Covid-19 entre otras son el síndrome de dificultad respiratoria aguda (SDRA), complicación más grave que comienza después del inicio de la disnea; a nivel cardíaco se describen arritmias, lesión cardíaca aguda, shock
ISSN 2773-7705

Periodo. Enero - Junio 2020

Vol. 2, Nro. 1, Publicado 2020-06-30

y miocardiopatía; de igual forma se puede manifestar tromboembolismo pulmonar o ictus; respuesta inflamatoria excesiva, similar al síndrome de liberación de citocinas con fiebre persistente, elevación de marcadores inflamatorios; por último, aunque poco frecuentes, se han descrito infecciones secundarias (9).

\section{Medidas Preventivas}

Actualmente, sin un tratamiento curativo o vacunas totalmente efectivas disponibles, la mejor estrategia colectiva para hacer frente al Covid-19 es controlar la fuente de infección. En este sentido, se ha demostrado que cumpliendo las medidas básicas de prevención de enfermedades, propuestas por la OMS, se puede lograr reducir el riesgo de transmisión. Entre estas, se encuentran (10):

Lavado de manos: Se debe adoptar el hábito de lavarse las manos con frecuencia, especialmente después del contacto directo con personas enfermas o su entorno. Es importante que el lavado se realice durante al menos 20 segundos y con la técnica adecuada que garantice la descontaminación de la piel en su totalidad.

Higiene respiratoria: Al toser o estornudar, la boca debe cubrirse con un pañuelo desechable y desecharse inmediatamente después de su uso, o con ropa (con el codo doblado) para evitar que el virus se propague. Además, se debe evitar tocarse los ojos, la nariz y la boca con las manos.

Separación física interpersonal: Se debe mantener una distancia entre personas de al menos 1,5 metros (preferiblemente 2 metros), ya que esta distancia se considera segura frente al posible desplazamiento de las gotitas de secreciones respiratorias antes de la sedimentación.

Descontaminación de superficies: después de que se describió que el SARSCoV-2 puede sobrevivir en las superficies durante algunas horas, la limpieza y desinfección frecuentes de las superficies es un medio de eliminación de la transmisión del virus (por ejemplo, pantallas de teléfonos celulares, teclados de computadoras, pasamanos, mesas, pomos de puertas, etc...), tanto en casa como en el trabajo.

Uso de mascarillas: A pesar de la controversia inicial, diversos estudios epidemiológicos han confirmado que el uso adecuado de mascarillas (cubriendo adecuadamente la nariz y la boca) es una de las medidas más efectivas.

Medidas de bioseguridad establecidas por el Ministerio de Salud Pública de Ecuador.

1. Se declaró Estado de Emergencia Sanitaria en todos los establecimientos del Sistema Nacional de Salud del Ecuador a través del acuerdo No.00126-2020 del Ministerio de Salud Pública, en los servicios de laboratorio, unidades de epidemiología, paramédicos, hospitalización y 


\section{HIGÍA DE LA SALUD}

consulta externa con el fin de evitar contagios masivos de COVID-19 (11).

2. Suspensión de la jornada laboral y las clases, además, se aplicaron medidas de distanciamiento social dictaminadas por el gobierno. Se prohibieron los eventos sociales en los meses de marzo y abril. Prohibición de transporte internacional e interprovincial de pasajeros.

3. Prohibición de reuniones sociales y funcionamiento de establecimientos comerciales con aforo de 30 personas, igualmente se restringió la circulación vehicular, permitida únicamente para actividades básicas y asuntos de salud.

4. Implementación del teletrabajo durante la emergencia sanitaria, tanto para empresas y organismos del sector público como para el sector privado.

5. El Ministerio de Inclusión Económica y Social (MIES), entregó del Bono de Protección Familiar por la Emergencia durante los meses de abril y mayo, así como entrega de kits de alimentación a todas las familias que se encontraron en situación vulnerable durante la crisis.

6. Prohibición de visitas en todos los centros geriátricos a escala nacional.

7. Restricción de movilización peatonal y vehicular, a excepción de compras urgentes de víveres y medicinas. (12).

8. Implementación de periodo de Aislamiento Preventivo Obligatorio (APO) para toda persona que llegara a Ecuador por cualquier vía.

9. El Ministerio de Salud Pública preparó 22 hospitales para atender casos de Covid-19, con especial atención a las poblaciones de atención prioritaria, habilitación de la línea telefónica 171 para personas con síntomas, uso de plataformas tecnológicas en telemedicina, y mayor control en los puntos de ingreso a territorio nacional (13). Como parte del proceso de enfrentamiento a la pandemia, el gobierno de Ecuador capacitó al personal multidisciplinario de salud para tener conocimientos de cómo tratar a infectados de COVID-19. Entre los métodos que se usaron para llevar a cabo esta capacitación se encontró:

Se emitió el documento "Consenso Multidisciplinario informado en la evidencia sobre el tratamiento de Covid-19" en el cual se explica brevemente el avance del virus en el Ecuador, qué procedimientos debe realizar el equipo multidisciplinario en el primer nivel de atención a infectados por el virus y qué clase de medicamentos se pueden utilizar para tratar a los pacientes (14).

Se emitió un documento dirigido al personal de enfermería sobre la atención a pacientes adultos con Covid-19 sin complicaciones respiratorias en el cual se explica el tratamiento que se debe realizar al momento de tratar a los pacientes mayores (15).
ISSN 2773-7705

Periodo. Enero - Junio 2020

Vol. 2, Nro. 1, Publicado 2020-06-30

Se realizaron comités en locales de salud, charlas informativas, conversatorios y reuniones virtuales con el personal de salud para dar a conocer sobre el Covid-19 (16).

Se habilitó una página en el sitio web del Ministerio de Salud en donde se ponen a disposición protocolos para tomar medidas contra el Covid-19, esto con el fin de que el personal multidisciplinario pueda tener conocimiento sobre cómo actuar en diversas situaciones tales como, manejo de cadáveres, tratamientos que se deben dar en aislamientos preventivos, test de pruebas rápidas, ingresos de viajeros al país (17)

En el ámbito de bioseguridad, se realizaron seminarios y capacitaciones, en los cuales se reforzó la protección del personal de salud, también se destacó el preparar los hospitales para la afluencia masiva de las personas, y en el uso correcto de los instrumentos médicos.

Uso de terapia complementaria para el tratamiento de infecciones respiratorias por coronavirus

Ficha del fitofármaco EQUINÁCEA ARKOPHARMA

Ingredientes activos, composición cuantitativa y grupos químicos o principios activos responsables de la actividad terapéutica del producto.

250 mg de raíz criomolida de Echinacea purpurea (L.) Moench (Equinácea). Como grupo químico responsable de la actividad terapéutica se encuentran las alquilamidas.

Artículos científicos que avalen la actividad farmacológica y toxicológica del producto o sus principios activos.

Los resultados obtenidos en un estudio muestran el efecto profiláctico de la Equinacea purpurea sobre las recidivas y la mejoría de los síntomas clásicos por la alteración en las afecciones del sistema inmunológico $(18 ; 19 ; 20)$. Un estudio con una formulación estandarizada de echinacea redujo los síntomas de personas con infección del tracto respiratorio (21).

\section{Clasificación}

farmacológica:

Inmonumodulador

Indicación terapéutica: Medicamento tradicional a base de plantas para el tratamiento de apoyo del resfriado común. Basado exclusivamente en su uso tradicional.

Forma farmacéutica: Cápsulas duras

Vía de administración: Oral

Dosificación: Adultos y adolescentes mayores de 12 años: tomar 2 cápsulas en el desayuno, 2 cápsulas en la comida y 2 cápsulas en la cena con un vaso de agua. El tratamiento deberá iniciarse con los primeros síntomas del resfriado. $\mathrm{Si}$ después de 10 días los síntomas persisten se deberá evaluar la situación clínica. 


\section{HIGÍA DE LA SALUD}

Efectos adversos conocidos: En raras ocasiones pueden aparecer

Toxicidad: No se reporta toxicidad

Contraindicaciones y advertencias:

Hipersensibilidad al principio activo o a alguno de sus excipientes incluidos en la familia de plantas Asteraceae.

Interacciones medicamentosas: No hay datos disponibles

Precauciones en el uso

El uso en niños menores de 12 años no está recomendado debido a que no existen datos médicos suficientes que avalen su seguridad.

En ocasiones pueden aparecer reacciones alérgicas en individuos sensibles o reacciones anafilácticas (22).

Modalidad de venta: libre

Datos de la planta que compone el producto

Nombre científico: Echinacea angustifolia

Nombres comunes: Equinácea

Características botánicas y etnomédicas de las especies incluidas en el producto

Es una planta perenne, herbácea, de la familia Asteraceae. Es originaria de los Estados Unidos de Norteamérica, se cree que lleva utilizándose desde hace siglos por las tribus indias de este continente para curar las heridas de las flechas o las picaduras de serpiente. Sus flores son muy similares a las margaritas, de tonos rosáceos o púrpuras, logra el metro de altura y su extensión llegar a ser de unos 45 centímetros.

Como principales usos tradicionales se describe que contribuye a disminuir la fiebre, la mucosidad y la tos asociadas tanto al resfriado como a otras enfermedades del sistema respiratorio. También tiene otra serie de

\section{Colaboradores de la Investigación:}

Espinoza Santos Mercedes Iveth; Flores Pico Concepción Jessenia; García Mejía Roberto Carlos; Holguín Serrano Karen Marisol; Lazcano Moreira Nayely Mariu; Bielka Pierina Loor Mero; López Saltos Jeniffer Stefany; Medina Aveiga Dayana Nicole; Mendoza Plaza Lorena Beatriz

\section{Bibliografía}

1 Gandhi R. T, Lynch J. B, Río C. 2020. Mild or moderate covid-19. The new england journal of medicine

[Online]; 2020. Acceso 13 de Agosto de 2020. Disponible en: https://www.nejm.org/doi/pdf/10.1056/NEJMcp2009249

2. MSP. [Online]; 2020. Acceso 13 de Agosto de 2020. Disponible en:

https://www.salud.gob.ec/el-ministerio-de-salud-publica-del-ecuador-msp-informasituacion-coronavirus/. 3. WHO [Online]; 2020. Acceso 15 de Agosto de 2020. Disponible en: https://www.who.int/emergencies/diseases/novel-coronavirus-2019/events-as-theyhappen.

4. Ambientalys. 2020. Información científica-técnica, COVID-19. [Online]; 2020. Acceso 15 de Agosto de 2020. Disponible en: https://www.ambientalys.com/informacion-cientifica-tecnica-covid.

5. Wölfel, R.; Corman VM, Guggemos, W.; Seilmaier, M.; Zange, S.; Müller, MA.; Niemeyer, D.; Jones,

TC.; Vollmar, P.; Rothe, C.; Hoelscher, M.; Bleicker, T.; Brünink, S.; Schneider, J.; Ehmann, R.;
Periodo. Enero - Junio 2020 procesos infecciosos, ya que actúa como como son: Infecciones vaginales, genitales o sífilis, malaria, migrañas e indigestión (23)

Pripales Constituyentes Químicos fenilpropanoides o derivados del ácido caféico, esencial, poliacetilenos, ácidos clorogénico y Partes utilizadas: Raíz

\section{Conclusiones}

que es una enfermedad altamente transmisible y la única metodología para prevenir la infección es evitar la exposición al virus, ya que en la específico para prevenir o tratar la infección por este virus. En lo que se refiere a las medidas de primordiales para prevenir la exposición a adoptar todas las medidas de bioseguridad complemento fundamental para la disminución de contagios. Como terapia complementaria a las coronavirus, se puede utilizar fitofármacos que respaldo científico en cuanto a su eficacia farmacológica. aplicaciones en su mayoría relacionadas con los 
ISSN 2773-7705

HIGÍA DE LA SALUD

Periodo. Enero - Junio 2020

Vol. 2, Nro. 1, Publicado 2020-06-30

Zwirglmaier, K.; Drosten. CH Y Wendtner, C. 2020. Virological assessment of hospitalized patients with COVID-2019 Nature. 581: 65-469 [Online]; 2020. Acceso 15 de Agostode 2020. Disponible en: https://doi.org/10.1038/s41586-020-2196-X.

6. Yan Bai; Lingsheng Yao; Tao Wei. 2020. Presumed Asymptomatic Carrier Transmission of COVID-19. JAMA. 2020; 323(14):1406-1407. [Online]; 2020. Acceso 15 de Agosto de 2020. Disponible en: https://jamanetwork.com/journals/jama/fullarticle/2762028.

7. Singhal, T. 2020. A Review of Coronavirus Disease-2019 (COVID-19) The Indian Journal of Pediatrics; 87: 281-286 [Online]; 2020. Acceso 16 de Agosto de 2020. Disponible en: https://doi.org/10.1007/s12098020-03263-6

8. Lu, R.; Zhao, X.; Li, J.; Niu, P.; Yang, B.; Wu, H.; Wang, W.; et al. 2020. Genomic characterisation and epidemiology of 2019 novel coronavirus: implications for virus origins and receptor binding. The lancet; 395(10224): 565-574. [Online]; 2020. Acceso 16 de Agosto de 2020. Disponible en: https://www.cdc.gov/coronavirus/2019-ncov/downloads/genomic-characterization-of-2019-nCoV-

Lancet-1-29-2020.pdf

9. Wichmann, D.; Sperhake, JP.; Marc Lütgehetmann, et al. 2020. Autopsy Findings and Venous Thromboembolism in Patients With COVID-19. Annals of internal medicine. [Online]; 2020. Acceso 19 de Agosto de 2020. Disponible en: https:/www.acpjournals.org/doi/full/10.7326/M202003? journalCode=aim

10. Organización Mundial de la Salud. 2016. Normas básicas de higiene del entorno en la atención sanitaria. Organización Mundial de la Salud. Ginebra. 54p

[Online]. Acceso 19 de Agosto de 2020. Disponible en:

https://apps.who.int/iris/bitstream/handle/10665/246209/9789243547237-

spa.pdf?sequence $=1 \&$ isAllowed $=\mathrm{y}$

11. Parlamento Andino. 2020. Principales medidas adoptadas por el gobierno ecuatoriano frente a la emergencia provocada por la COVID-19 [Online]. Acceso 14 de Agosto de 2020. Disponible en: https://parlamentoandino.org/wp-content/uploads/2020/10/Principales-medidas-adoptadas-por-elgobierno-ecuatoriano.pdf

12. Ministerio de Transporte y Obras Públicas. 2020. Con acuerdo ministerial se precisa la aplicación de las restricciones para el transporte [Online]; 2020. Acceso 15 de Agosto de 2020. Disponible en: https://www.obraspublicas.gob.ec/con-acuerdo-ministerial-se-precisa-laaplicacion-de-las-restriccionespara-el-transporte/.

13. Secretaría Técnica Plan Toda una Vida.2020. Emergencia Sanitaria por COVID-19 incorpora medidas de prevención para grupos de atención prioritaria [Online]. Acceso 15 de Agosto de 2020. Disponible en: https://www.todaunavida.gob.ec/emergencia-sanitaria-por-covid-19-incorpora-medidasde-prevencionpara-grupos-de-atencion-prioritaria/.

14. Ministerio de Salud Pública. 2020. Consenso Multidisciplinario informado en la evidencia sobre el tratamiento de Covid-19 [Online]; Acceso 16 de Agosto de 2020. Disponible en: https://www.salud.gob.ec/wp-content/uploads/2020/08/Consenso-Multidisciplinario-informado-en-laevidencia-sobre-el-tratamiento-de-Covid-19-V9_11_08_2020_compressed.pdf 
ISSN 2773-7705

HIGÍA DE LA SALUD

Periodo. Enero - Junio 2020

Vol. 2, Nro. 1, Publicado 2020-06-30

15. Ministerio de Salud Pública. 2020. Atención de enfermería a pacientes adultos con COVID-19 sin complicaciones respiratorias. Protocolo. Quito: Ministerio de Salud Pública, Dirección Nacional de Normatización de Talento Humano en Salud, Dirección Nacional de Normatización Federación Ecuatoriana de Enfermeras y Enfermeros. [Online]. Acceso 16 de Agosto de 2020. Disponible en: https://www.salud.gob.ec/wp-content/uploads/2020/08/PROTOCOLO-COVID-19-enfermer\%C3\%ADa.pdf

16. Ministerio de Salud Pública. 2020. Comités Ciudadanos Locales de Salud - Proceso de Formación Covid-19. [Online]; 2020. Acceso 16 de Agosto de 2020. Disponible en: https://www.salud.gob.ec/comitesciudadanos-locales-de-salud-proceso-de-formacioncovid-19/\#.

17. Ministerio de Salud Pública. 2020. Documentos Normativos Coronavirus Ecuador. [Online]; 2020. Acceso 16 de Agosto de 2020. Disponible en: https:/www.salud.gob.ec/documentos-normativos-covid-19ecuador/.

18. Márquez LR. 2000. La equinàcea purpùrea. Revista de Fitoterapia; I (1):15-24.

Disponible

en:

https://www.fitoterapia.net/php/descargar_documento.php?id=4817\&doc_r=sn\&num_volumen=1\&secc_ volumen $=5951$

19. Barnes, J.; Anderson, L. A.; Gibbon, S y Phillipson, JD. 2005. Echinacea species (Echinacea angustifolia (DC.) Hell., Echinacea pallida (Nutt.) Nutt., Echinacea purpurea (L.) Moench): a review of their chemistry, pharmacology and clinical properties. 57:929-954. https://onlinelibrary.wiley.com/doi/epdf/10.1211/0022357056127

20. Wagner, H.; Stuppner, H.; Schäfer, W.; ZenkImmunologically, M. 1998. Active polysaccharides of Echinacea purpurea cell cultures. Phytochemistry 27(1):119-126

21. Goel, V.; Lovlin, R.; Barton, R.; Lyon, M. R.; Bauer, R.; Lee, T. D. G y Basu, T. K. Efficacy of a standardized Echinacea preparation (Echinilin) for the treatment of the cold. Journal of Clinical Pharmacy and Therapeutics. 2004; 29(1):75-83.

22. Agencia española de medicamentos y productos sanitarios. 2015. Ficha Técnica Equinacea Arkopharma [Online]. Acceso 2 de Septiembre de 2020. Disponible en: https://cima.aemps.es/cima/dochtml/ft/72921/FichaTecnica_72921.html

23. Cebrian J. 2019. 6 plantas medicinales que puedes cultivar en tu jardín. Fitoterapia. Cuerpomente. [Online]; 2019. Acceso 17 de Agosto de 2020. Disponible en: https://www.cuerpomente.com/saludnatural/terapias-naturales/que-plantasmedicinales-se-pueden-cultivar-jardin-casa_4532/3.

24. Loaiza, J; Valverde, R; Rodríguez, G; Molina, J. 2004. Análisis cuantitativos de los principales constituyentes químicos de raíces de Echinacea purpurea y E. Angustifolia producidas en Costa Rica. Agronomía Costarricense. 28(2):53-59. 